\title{
Trombectomía microquirúrgica de rescate para accidente cerebrovascular isquémico
}

\section{Microsurgical thrombectomy as recue therapy for acute ischemic stroke}

\author{
José Luis Cuevas Seguel', Jorge Tabilo Sepúlveda' \\ ${ }^{1}$ Neurocirujano. Servicio de Neurocirugía, Hospital de Puerto Montt, Chile.
}

\begin{abstract}
Resumen
El tratamiento del accidente cerebrovascular isquémico agudo (ACV) ha evolucionado significativamente en los últimos años. Aunque la trombolisis endovenosa con t-PA sigue siendo un pilar terapéutico, desde 2015 la trombectomía mecánica endovascular (TE) se ha establecido como el nuevo estándar de manejo debido a los promisorios resultados obtenidos en diversos ensayos clínicos. Pese a ello, el acceso a esta terapia sigue siendo limitado en gran parte del mundo. La trombectomía microquirúrgica (TM) puede ser una alternativa terapéutica de rescate para aquellos pacientes con oclusiones de grandes arterias en quienes la terapia trombolítica es superada o no exista disponibilidad de TE. En este reporte, presentamos un caso-vídeo de una TM realizada en un paciente de 55 años con oclusión de arteria cerebral media (ACM) izquierda, en quien se intentó terapia trombolítica sin éxito y no se dispuso de terapia endovascular. La cirugía se realizó luego de 3 horas de iniciados los síntomas mediante un acceso pterional izquierdo, tras comprobar mediante angioTC la persistencia de la oclusión arterial post trombolisis. El vídeo ilustra el análisis pre-quirúrgico del caso, la apertura de la fisura silviana, exposición arterial y trombectomía, enfatizando los aspectos técnicos relevantes, así como la efectividad y seguridad de la cirugía.
\end{abstract}

Palabras clave: Cerebral revascularization, stroke, thrombectomy, microsurgery, video.

Video disponible en:

https://youtu.be/a92Bq-bpuXA

Conflicto de interés:

El autor no declara conflicto de interés.

\section{Correspondencia a:}

Dr. José Luis Cuevas

Servicio de Neurocirugía Hospital de Puerto Montt.

Los Aromos 65, Puerto Montt, Chile.

cuevasseguel.joseluis@gmail.com 